\title{
First experiences with genetic counselling based on predictive DNA diagnosis in hereditary glomus tumours (paragangliomas)
}

Jan C Oosterwijk, Jeroen C Jansen, Evert $M$ van Schothorst, Annemiek W Oosterhof, Peter Devilee, Egbert Bakker, Moniek W Zoeteweij, Andel G L van der Mey

\begin{abstract}
Hereditary glomus tumour (MIM 168000) or paraganglioma (PGL) is a slowly progressive disorder causing benign tumour growth predominantly in the head and neck region. Though benign in nature the tumours can lead to severe morbidity. Inheritance of PGL is autosomal dominant and is strongly modified by genomic imprinting; only a paternally transmitted PGL gene leads to symptoms. A gene for PGL has recently been mapped to 11 q22.3q23.
\end{abstract}

Genetic counselling on the basis of DNA linkage diagnosis was offered in an extended Dutch pedigree. Thirty-two subjects opted for further counselling, of whom 20 applied for DNA testing and participated in a standardised protocol. Sixteen cases had presymptomatic testing (paternal allele); four of these appeared to have the at risk haplotype and in two of them a glomus tumour was subsequently detected on MRI. In one case linkage results were inconclusive (recombination) and one person did not want to learn his test result. Four cases had testing for carrier status (maternal allele) of which one appeared to be a carrier.

Our data show that genetic counselling gains significant accuracy when based on parent of origin, sex of the counsellee, and DNA linkage diagnosis. Moreover, a normal DNA result may prevent unnecessary worry and investigations, while an established presymptomatic diagnosis will guide adequate clinical management. The psychological impact of counselling and predictive DNA testing is unclear as yet. Further investigations into the natural history of PGL in gene carriers and into the psychological impact of DNA testing is desirable

(f Med Genet 1996;33:379-383)

Key words: paragangliomas; predictive testing; genomic imprinting.

Tumour growth in the paraganglia tissue of the head and neck (for example, carotid bodies, jugular bodies, vagal bodies) is a rare disorder affecting around 1 in 100000 people. ${ }^{12}$ Pooled Dutch data from pathological laboratories show a mean incidence of 17 (operated) cases a year (1986-1992) which corresponds to approximately $0 \cdot 1$ in $100000 .^{3}$ The Dutch Na- tional Cancer Registry registered 90 glomus tumours over a 10 year period. ${ }^{4}$

Symptoms usually occur between 15 and 45 years of age and consist of local swelling and cranial nerve injury and are rarely associated with vasoactivity as in phaeochromocytomas. The percentage in which malignant degeneration occurs is unclear but may be between 4 and $16 \%$. Metastases are seen in less than $5 \%$ and multiple tumours in around $5 \% .^{5-8}$ Although benign in nature, tumour growth is often progressive and can severely affect surrounding structures. This may lead to disfiguring local swellings and give rise to cranial nerve injury leading to facial asymmetry, deafness, or hoarseness. In more severe cases the base of the skull can be involved. In general the therapy of choice is surgery, which is usually possible without major complications for small tumours. However, the safety of surgical intervention depends, among other things, on the size and localisation of the glomus tumour(s); vascular and neurological complications are frequent in more advanced cases. It is difficult, however, to forecast tumour growth behaviour and in many cases "wait and see" could be an alternative approach, since life expectancy in affected patients is not significantly reduced. ${ }^{89}$ Periodic screening of patients using MRI, CT scan, or angiography is the only way to detect early, symptom free growth of glomus bodies in order to be able to intervene at an early stage or to monitor the usually indolent growth pattern. ${ }^{10}$

In hereditary glomus tumours or paragangliomas (PGL) the growth of glomus bodies is predominantly in the head and neck region. PGL presumably forms a small fraction of all cases with glomus body tumours; according to published reports some 5 to $10 \%$ of cases are genetic in origin. ${ }^{68}$ This figure may actually be substantially higher given the fact that generations are often skipped and the clinical picture is not always recognised properly, as explained below. In familial cases multiple tumours, both synchronous and metachronous, occur in at least $30 \%{ }^{68}$ Transmission of PGL is autosomal dominant with highly variable expressivity and reduced penetrance. The inheritance pattern has long been puzzling clinicians owing to a very reduced penetrance. ${ }^{11-13}$ Van der Mey et $a l^{14}$ postulated that the inheritance pattern is compatible with modification of gene expression by genomic imprinting. Only a paternally transmitted PGL gene leads to symptoms, irrespective of the 


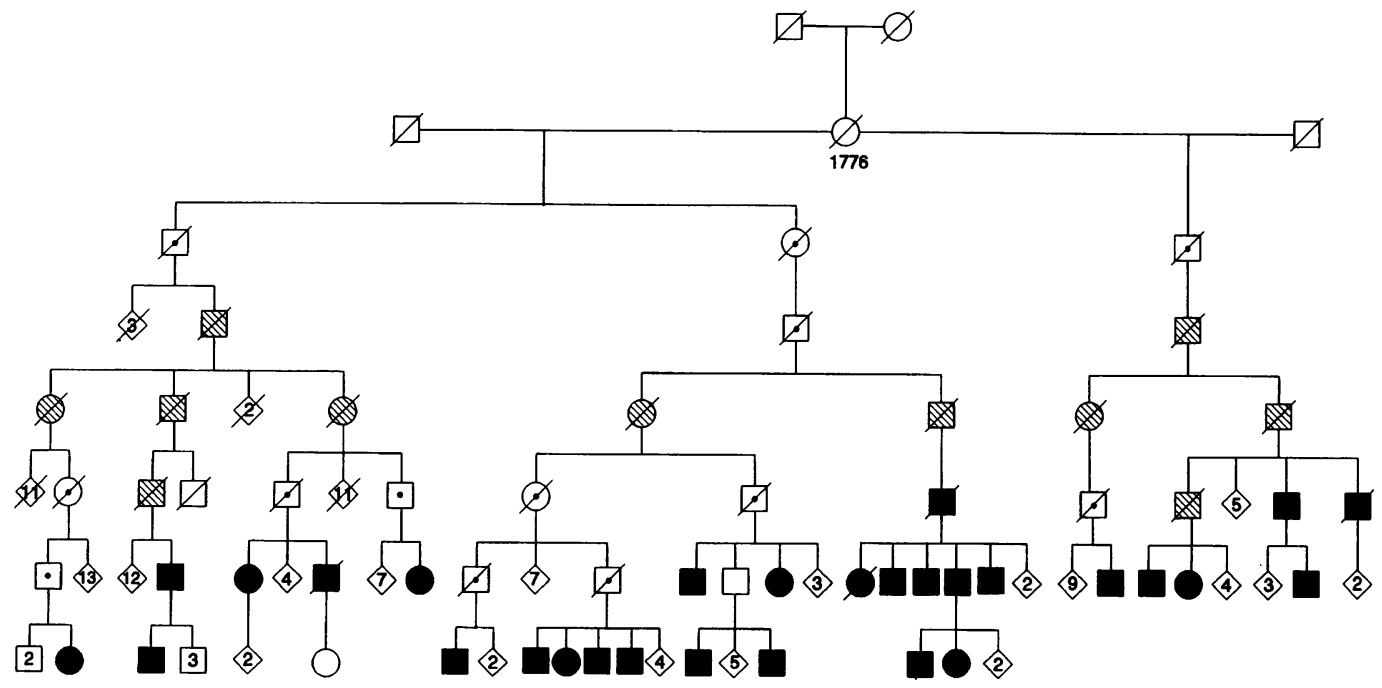

Extended pedigree of the Leiden PGL family. Genealogical data lead to one common founder for several family branches.

clinical status of the father, whereas a maternally derived PGL gene (irrespective of the clinical status of the mother) gives rise to carriership without symptoms. This explanation, known to occur in other neoplastic disorders, ${ }^{15}$ was later supported by statistical analysis in Dutch families. ${ }^{1617} \mathrm{McC}$ caffrey et $a l^{9}$ recently reported genomic imprinting in a number of PGL pedigrees ascertained in the USA.

Hereditary PGL is relatively frequent in the area of Leiden, The Netherlands, owing to a founder effect segregating in several relatively isolated villages. ${ }^{818} \mathrm{~A}$ gene for PGL has recently been mapped by linkage analysis to $11 \mathrm{q} 22.3$ q23. ${ }^{161719}$ This enables DNA diagnosis based on flanking markers in families where PGL is linked to this locus. Combining the correct interpretation of genomic imprinting with DNA diagnosis using flanking markers will greatly improve counselling accuracy for PGL. Given the implications of being at risk for PGL (periodic screening, multiple tumours, early surgical intervention) and given the genetic nature of the disorder, early establishment of the genotype may be useful both for clinical and genetic counselling. Moreover, since predictive DNA testing may have significant psychological impact, this aspect of counselling needs to be assessed. We report here our experiences of genetic counselling in 32 persons of whom 20 were DNA tested according to a fixed protocol. The data on psychological aspects and follow up will be published in the near future.

\section{Patients and methods}

The patients are members of an extended Dutch pedigree in which linkage research was performed previously (figure). Linkage to 11q22.3-q23 between markers STMY and CD3D was established firmly with a lod score of over 6.5 at $\theta=0^{1617}$ as well as haplotype sharing for D11S938 to D11S908 in all patients. ${ }^{19}$ Genetic counselling based on imprinting data and DNA linkage using a fixed protocol was offered to the family members by means of a letter from the ENT department. This letter was sent to several key persons in the family who distributed the information further. Of the approximately 90 persons in this kindred that may have received the letter, 68 members responded (written consent), of whom $32(47 \%)$ opted for further counselling. The people who opted for DNA testing were counselled according to a certain protocol and were asked to participate in a psychological study consisting of several questionnaires and sessions with a psychologist before and after DNA testing. The design of the study was approved by the local ethical committee.

\section{COUNSELLING PROTOCOL}

The counselling procedure was designed as a multidisciplinary approach involving the Departments of Clinical Genetics, Human Genetics, and Otorhinolaryngology (table 1). At the first session the family histories were recorded and the clinical picture of PGL and the mode of inheritance was explained. Furthermore the procedure of DNA testing was explained and a prior risk was established using age dependent expression data of Jansen et $a l,{ }^{18}$ based on the documented age at first symptoms in 77 familial cases known to the ENT Department. When possible we aimed at testing key persons high in the pedigree (for example, a parent at risk) in order to avoid unnecessary testing (for example, in all children) and to prevent disclosure of unwanted information (for example, testing a $25 \%$ at risk person may disclose a parental haplotype). Subsequently the psychologist (AO, MZ) explained the psychological protocol consisting of two pretest and two post-test sessions and several psychological questionnaires. ${ }^{20-22}$ When the counsellee did not wish to participate in the scientific part of the protocol this did not

\section{Table 1 Timetable of counselling procedures}

\begin{tabular}{ll}
\hline 1 & Intake and first session with psychologist \\
$2(4-6 w k)$ & Blood sampling and second session with psychologist \\
$3(10-12 w k)$ & Disclosure of test result (clinical geneticist) \\
$4(11-12 w k)$ & Third session with psychologist \\
$5(40-42$ wk) & Fourth session with psychologist \\
\hline
\end{tabular}


Table 2 Description of 20 cases where DNA diagnosis was performed using flanking markers

\begin{tabular}{|c|c|c|c|c|c|}
\hline Case & Sex & Age (y) & Prior risk (\%) & Result & Follow up ENT \\
\hline \multicolumn{6}{|c|}{ Presymptomatic testing (paternal allele) $(n=16)$} \\
\hline $1-17$ & $\mathbf{M}$ & 28 & 35 & neg & \\
\hline $1-61$ & $\mathbf{M}$ & 34 & 30 & pos & Scan + \\
\hline $1-62$ & $\mathbf{M}$ & 33 & 30 & neg & \\
\hline $1-63$ & $\mathbf{M}$ & 26 & 35 & pos & Scan - \\
\hline $1-99$ & $\mathrm{~F}$ & 58 & 20 & neg & \\
\hline $1-64$ & $\mathbf{M}$ & 71 & 20 & withdrew & \\
\hline $1-104$ & $\mathbf{M}$ & 34 & 30 & not informative & Scan - \\
\hline $1-78$ & $\mathbf{M}$ & 66 & 20 & neg & \\
\hline $1-a$ & $\mathbf{M}$ & 28 & 35 & pos & Scan + \\
\hline $1-113$ & $\mathrm{~F}$ & 24 & 40 & neg & \\
\hline $1-b$ & $\mathbf{M}$ & 23 & 40 & neg & \\
\hline $1-c$ & $\mathbf{M}$ & 18 & 45 & neg & \\
\hline $9-47$ & $\mathrm{~F}$ & 33 & 30 & neg & \\
\hline $8-40$ & $\mathbf{M}$ & 45 & 25 & neg & \\
\hline $8-15$ & $\mathbf{F}$ & 39 & 25 & pos & Pending \\
\hline $8-16$ & $\mathrm{~F}$ & 37 & 30 & neg & \\
\hline \multicolumn{6}{|c|}{ Carrier testing (maternal allele) $(n=4)$} \\
\hline $1-34$ & $\mathrm{~F}$ & 61 & 50 & neg & \\
\hline $1-40$ & $\mathrm{~F}$ & 64 & 50 & pos & \\
\hline $1-117$ & $\mathrm{~F}$ & 43 & 50 & neg & \\
\hline $9-88$ & $\mathbf{M}$ & 70 & 50 & neg & \\
\hline
\end{tabular}

affect the routine counselling procedure and psychological support.

At the second appointment, after four to six weeks, a blood sample was taken (JCO) for DNA linkage analysis, and a second session took place with the psychologist. Some eight weeks later the DNA test results were discussed with the counsellee. Approximately six months after disclosure of the test result there were follow up sessions with the psychologist.

Patients who appeared to be carriers of a paternally derived gene were offered further counselling by the ENT surgeon (AGLvdM). They were instructed about the signs and symptoms of PGL, and physical examination (repeated once a year) and an MRI scan (repeated once every two years) was offered.

\section{MOLECULAR ANALYSIS}

DNA linkage analysis was performed using flanking polymorphic markers. From counsellees who opted for presymptomatic DNA diagnosis or DNA carrier testing, $20 \mathrm{ml}$ EDTA blood was taken (JCO). In several cases blood from the unaffected parent was also taken (JCO) in order to establish the phase of the haplotypes. The blood was directly transported to the laboratory and all analyses were performed according to national diagnostic quality standards. The haplotype analysis was checked with the former research data of the extended pedigree whenever present (15 cases). Haplotype analysis was based on (from centromere to telomere) D11S527, D11S876, D11S927, D11S897, D11S560, D11S938, D11S1327, D11S1340, APOC3, D11S490, CD3D, and D11S836. ${ }^{2324}$ More markers were used when the above mentioned were uninformative, for example D11S908, D11S939, D11S2082. The distance between the informative markers flanking the PGL region was between approximately 2 and $7 \mathrm{cM}$ in all cases. ${ }^{19}$

\section{Results}

Sixty-eight people returned the form in which they could indicate whether they wanted further counselling or not. Thirty-six people refrained from further genetic counselling and DNA analysis. Five of these were clinically affected with PGL, 10 were potential carriers of a maternally derived allele, four were at risk of PGL (paternal allele), and in 17 their status was unknown.

Thirty-two out of 68 people (47\%) opted for genetic counselling. Five patients were clinically affected and received extensive genetic counselling without the need for molecular diagnosis. The remaining 27 persons were not affected as far as they knew; 20 were at risk for a paternal PGL allele and seven for a maternal allele. Of these 27 cases, 23 were seen at the Department of Clinical Genetics, and the remaining four cases learned their result in an indirect way because their parent at risk was among the people tested and proved not to carry the PGL gene.

In the 23 symptom free cases seen, the closest affected relative according to family history was first degree in 18 cases, second degree in three cases, and third degree in two cases. Deafness and facial palsy/asymmetry are the symptoms of PGL that counsellees refer to as the clinical picture, but hoarseness, postsurgical mutilation, and death are also mentioned. The perception of the severity of PGL depended on the clinical picture of known affected relatives.

Of the 23 symptom free cases, three counsellees (two at disease risk, one potential carrier) refrained from further investigations after the intake session. In 20 symptom free cases, DNA linkage diagnosis has been performed (table 2). For all these cases a prior risk of carrying the PGL gene was determined (table 2 ). For those at risk of a paternally derived gene we used the age at first symptom curve as determined in the Leiden patient set by Jansen et al. ${ }^{18}$

Of the 20 DNA diagnoses in 16 cases this concerned presymptomatic testing (paternal transmission). Four cases appeared to be gene carriers, 10 are non-carriers, one DNA result is inconclusive owing to a recombination event, and one person refrained from learning the test result. This man feared he could not cope with the feelings of guilt towards his children if he turned out to be a gene carrier. The four positive results increased the prior risk for four children to $50 \%$, two potential maternal and two potential paternal transmissions. The eight negative results provided a result for over 11 children who were in a $25 \%$ at risk situation that now changed to $<0 \cdot 1 \%$, that is the population risk of isolated PGL. This concerned a potential paternal transmission in eight cases and a potential maternal transmission in three cases.

In four cases DNA diagnosis was performed for carrier testing (maternal transmission). In these cases their actual age does not affect the prior chance of carriership. One person appeared to be a gene carrier, which increased the prior risk of four children to $50 \%$ of inheriting a maternal allele. Three cases were non-carriers, two female and one male. This made the risk of inheriting a maternal allele 
for eight children and a paternal allele for six children negligible.

Four counsellees, three who received a positive DNA result and the one case with an uninformative DNA result, visited the ENT outpatients clinic for further counselling (table 2). They were examined physically by the ENT surgeon and had an MRI investigation. Two out of three showed a tumour of the glomus caroticum (one of them, 1-61, was operated upon), one was negative on MRI. The case with the recombination had a normal MRI five years ago and now again shows no abnormalities on MRI.

\section{Discussion}

Paraganglioma is a relatively benign disorder leading to tumour growth in the head and neck region. According to published reports, at least $5 \%$ is hereditary; in our geographical region more than $50 \%$ appear familial and in these families genetic counselling should be offered. It is our experience that thorough family history data are needed before it can be concluded that a case is sporadic, because of the sometimes very mild symptoms in key relatives or the skipping of generations owing to genomic imprinting or both. This is illustrated by the fact that of our 20 people with a risk of $50 \%$ (at birth) of inheriting the PGL gene, two have a third degree and three have a second degree relative as the closest relative known to be clinically affected. Moreover, as in many other well known genetic tumour susceptibility syndromes, sporadic PGL cases with multiple tumours are suspected to be the result of an inherited mutation. ${ }^{25}$

Of the approximately 90 people in this pedigree that may have received our letter describing the possibility of DNA based genetic counselling, 68 people responded. Of these, 32 $(47 \%)$ opted for further counselling. Since the informing letter was forwarded by key persons in the pedigree we do not know the exact number of people who actually received this information. If all 90 were reached and nonresponders are counted as negative responders, then the uptake is 32 out of 90 (35\%). If only 68 people were reached and all responded, the uptake would be $47 \%$. This means that in this family the uptake of genetic counselling is somewhere between 35 and $47 \%$. This is still significantly higher than the uptake of 10 to $15 \%$ in Huntington's disease, ${ }^{26}$ a disorder where predictive testing offers no preventive or therapeutic consequences for the counsellee. The percentage is lower, however, than in familial adenomatous polyposis where it is some $90 \%,{ }^{27}$ or in MEN2A (some $95 \%$ ), ${ }^{28}$ where the preventive options are evident. Both disorders mentioned are potentially lethal in contrast to PGL, so the relative mildness of the disorder might also be a reason for the lower uptake.

The population that opted for counselling and testing did not differ significantly from those that responded negatively with regard to age and gender (data not shown). In the first group, five were affected with PGL, 20 were at risk for paternal transmission, and seven were at risk for maternal transmission. In the second group, five were affected, four were at risk for a paternal allele, 10 were at risk for a maternal allele, and in 17 cases the status was unknown. There seems to be a trend that being at risk of developing PGL (paternal transmission) is more often a motivation for testing than merely carriership.

DNA diagnosis in all counsellees was performed against the background of extensive research linkage data of the same extended family. This made diagnostic linkage analysis relatively easy and excluded the problem of genetic heterogeneity. The latter is important since the issue of whether there are two different loci for PGL on the long arm of chromosome 11 segregating in The Netherlands is still unresolved. ${ }^{2930}$ In our extended family, linkage to $11 \mathrm{q} 13$ was excluded. ${ }^{17}{ }^{19}$ Of $20 \mathrm{DNA}$ analyses performed, only one case turned out to be uninformative owing to a recombination event in the PGL region. In all other cases the DNA result had a certainty of over $99.9 \%$ since the chance of a double recombinant between the PGL locus and both flanking markers is very low. ${ }^{19}$

It is evident that genetic counselling and risk estimation are much more specific when based on parental origin of the PGL gene and on DNA diagnosis using flanking polymorphic markers. For people at risk of a paternal PGL gene the reliability of a DNA diagnosis performed once is age independent, less burdensome than (regular) MRI or angiography, and less expensive than most other procedures. DNA diagnosis is in fact the only way a person at risk for a maternal PGL gene can learn his or her status, unless the disease has already manifested itself in affected offspring. This DNA carrier diagnosis for an autosomal dominant disorder with no consequences for the "patient's" health, even at a young age, is unusual in clinical genetics. It will be interesting to compare the results of the psychological study ${ }^{22}$ with research findings on the detection of a BRCA1 or BRCA2 mutation in males. Data are scarce on the uptake and (psychological) implications in this category, but feelings of (survivor) guilt might be anticipated. ${ }^{2131}$

Four patients appeared to be a carrier of a paternal allele at the ages of $26,28,34$, and 39 respectively. In two of them a glomus tumour was subsequently detected on MRI, and one has been operated upon recently. The fourth is awaiting MRI analysis. In all paternal gene carriers an MRI investigation is valuable in order to make comparisons with subsequent imaging possible. Moreover, since glomus tumours can be removed more safely at an early stage, it is expected that regular MRI scanning on the basis of the proven presence of a paternal PGL gene will improve patient management by the ENT surgeon.

The fact that $75 \%$ of DNA tests proved to be negative is unexpected given an autosomal segregation ratio of $50 \%$. This may be explained by the fact that five symptomatic counsellees were excluded from DNA testing because their status was evident clinically. 
Moreover, all persons tested for a paternal allele were adult (mean age 37 years) and so their prior risk was decreased (mean risk $28 \%$ ) because of their age, given the age related penetrance of PGL. The mean prior risk of $28 \%$ fits well with the $25 \%$ positive test results.

The psychological impact of genetic counselling and predictive DNA testing for PGL has not been described as yet and it is unknown how people at risk cope with their test results. One might expect that the parental origin of the gene may affect psychological impact. Apart from comparison with other late onset disorders, a comparison with certain types of carriership would be interesting. Also the preventive and therapeutic aspects of PGL need to be taken into account when comparing counselling data with other late onset disorders. Preliminary data show that the main reason for DNA testing is for the sake of the children, and this applies especially to the older people, whose risk of still developing clinically relevant glomus tumours is negligible. These and other physiological issues are currently being investigated and will be published in the future. ${ }^{22}$

\section{Conclusion}

Since the genetic and clinical aspects (instruction, regular examination, treatment, and prognosis) of PGL are rather complex and closely related we suggest that a multidisciplinary approach is desirable in counselling hereditary PGL. Our experiences to date show that genetic counselling gains significant accuracy when based on parent of origin, sex of the counsellee, and DNA linkage diagnosis. Moreover, a normal DNA result may prevent unnecessary worry and investigations, while an established presymptomatic DNA diagnosis will guide clinical management. Further investigation into the natural history of PGL in gene carriers and into the psychological impact of DNA testing is desirable.

We thank Alfons F J Bardoel and Yvonne Zoet for assisting with DNA analysis, Cees J Cornelisse and Lodewijk Sandkuyl for helpful discussions, and especially Ank Boukens for secretarial assistance.

1 Lack EE, Cubilla AL, Woodruff JM, Farr HW. Paragangliomas of the head and neck region. A clinical study in 69 patients. Cancer 1977;39:397-409.

2 Lack EE, Cubilla AL, Woodruff JM. Paragangliomas of the head and neck region. A pathologic study of tumors from 71 patients. Hum Pathol 1979;10:191-218.

3 PALGA Foundation, Amsterdam 1995.

4 SIG Foundation, Utrecht, 1990.

5 Batsakis JG. In: Tumors of the head and neck. 2nd ed. Baltimore: Williams \& Wilkins, 1979:369-80.
6 Gufferman S, Gillman MW, Paternak LR, Peterson CL, Young WG. Familial carotid body tumors: case study and epidemiologic review. Cancer 1980;46:2116-22.

7 Meyer FB, Sundt TM, Pearson BW. Carotid body tumors: a subject review and suggested surgical approach. $f \mathrm{Ne}$ a subject review and sugge

8 Van der Mey AGL. Head and neck paragangliomas. Thesis, Leiden University, 1992.

9 McCaffrey TV, Meyer FB, Michels VV, Piepergras DG, Marion MS. Familial paragangliomas of the head and neck. Arch Otolaryngol Head Neck Surg 1994;120:1211-6.

10 Van Gils APG, van der Mey AGL, Hoogma RPLM, et al. MRI screening of kindred at risk of developing paragangliomas: support for genomic imprinting in hereditary glomus tumours. $B r \mathcal{F}$ Cancer 1992;65:903-7.

11 Bartels J. De tumoren van het glomus jugulare. Thesis, Gronigen University, 1949.

12 Van Baars F, Cremers C, van den Broek P, Geerts S, Veldman $\mathrm{J}$. Genetic aspects of nonchromaffin paraganglioma. Hum Genet 1982;60:305-9.

13 Van Baars, FM, Cremers CWRJ, van den Broek P, Veldman JE. Familial non-chromaffinic paragangliomas (glomus JE. Familial non-chromaffinic paragangliom
tumors). Acta Otolarnyngol 1981;91:589-93.

14 Van der Mey AGL, Maaswinkel-Mooy PD, Cornellisse CJ, Schmidt PH, van de Kamp JJP. Genomic imprinting in hereditary glomus tumours: evidence for new genetic theory. Lancet 1989;ii:1291-4.

15 Ferguson-Smith AC, Reik W, Surani A. Genomic imprinting and cancer. Cancer Surv 1990;9:487-503.

16 Heutink P, van der Mey AGL, Sandkuijl L, et al. A gene subject to genomic imprinting and responsible for hereditary paragangliomas maps to chromosome 11q23-qter Hum Mol Genet 1992;1:7-10.

17 Heutink P, van Schothorst EM, van der Mey AGL, et al. Further localization of the gene for hereditary paragangliomas and evidence for linkage in unrelated families. Eur 7 Hum Genet 1994;2:148-58.

18 Jansen JC, van Schothorst EM, van der Mey AGL, et al. Hereditary paragangliomas. Epidemiological and genetic aspects (in press)

19 van Schothorst EM, Jansen JC, Bardoel AFJ, et al. A 2cM interval on 11q22-q23, containing PGL, an imprinted gene responsible for hereditary paragangliomas Eur $\mathcal{F H u m}$ Genet (submitted).

20 Tibben A, Vegter-van der Vlis M, Skaastad MI, et al. DNAtesting for Huntington's disease in the Netherlands, a retrospective study on psychological effects. Am $\mathcal{F}$ Med Genet 1992;44:94-9.

21 Tibben A. What is knowledge but grieving? Thesis, Rotterdam University, 1993.

22 Oosterhof AW, Zoeteweij MZ, Bakker E, et al. Predictive DNA testing for hereditary glomus tumours (PGL); psychological implications of genomic imprinting (in press).

23 NIH-CEPH Collaborative Mapping Group. A comprehensive genetic linkage map of the human genome. prehensive genetic linkage
Science 1992;258:67-102

24 Guyapay G, Morissette J, Vignal A, et al. The 1993-94 Généthon human genetic linkage map. Nature Genet 1994; 7:246-339.

25 Eng C, Stratton M, Ponder B, et al. Familial cancer syndromes. Lancet 1994;343:709-13.

26 Van der Steenstraten IM, Tibben A, Roos RAC, et al. Predictive DNA-testing for Huntington disease: nonparticipants compared with participants in the Dutch program. Am F Hum Genet 1994;55:618-25.

27 Van Leeuwen-Cornelisse I. Foundation for detection of hereditary tumors. Leiden, The Netherlands, 1995.

28 Sijmons RH, Sikkens EH, Grosfeld FJM. Presymptomatic DNA testing in MEN2A families: practical counselling issues. In: Muller HJ, Weber W, eds. Proceedings of $2 n d$ international research conference on familial cancer. Basel: international rese

29 Mariman ECM, van Beersum SEC, Cremers CWJ, van Baars FM, Ropers HH. Analysis of a second family with hereditary non-chromaffin paragangliomas locates the underlying gene at the proximal region of chromosome 11q. Hum Genet 1993;91:357-61.

30 Mariman ECM, van Beerseum SEC, Cremers CWRJ, Struycken PM, Ropers HH. Fine mapping of a putatively imprinted gene for familial non-chromaffin paragangliomas to chromosome 11q13.1: evidence for genetic heterogeneity. Hum Genet 1995;95:56-62.

31 Dudok de Wit AC, Tibben A, Frets PG, et al. Predictive DNA-testing in the Netherlands for hereditary breast and ovarian cancer (HBOC): a case study (in press). 